\title{
Evolution of EEG findings in children with autism spectrum disorder: A tertiary care centre's clinical experience
}

\author{
Drew M Thodeson ${ }^{1 *}$, Delaney Dowd ${ }^{2}$, Sailaja Golla ${ }^{1}$, Patricia Evans ${ }^{1}$, Rong Huang ${ }^{3}$ and Deepa Sirsi ${ }^{1}$ \\ ${ }^{1}$ Department of Pediatrics and Neurology and Neurotherapeutics, Division of Child Neurology, UT Southwestern Medical Center, Dallas, TX 75390, USA \\ ${ }^{2}$ UT Southwestern School of Medicine, UT Southwestern Medical Center, Dallas, TX 75390, USA \\ ${ }^{3}$ Children's Medical Center Research Institute, Children's Medical Center Dallas, TX 75235, USA
}

\begin{abstract}
Epilepsy and Autism Spectrum Disorder (ASD) are frequently co-morbid conditions. There are no clear testing modalities or biomarkers to distinguish children with ASD who are at risk for developing epileptic seizures from those who are not. Epileptiform electroencephalogram (EEG) abnormalities are common in children with ASD, suggesting a possible shared underlying pathophysiology with epilepsy. Our study analysed EEGs in children with ASD who underwent serial EEG studies. We show that initial EEG classification was a significant prognosticator for future EEG findings ( $<<0.0001)$. Our study failed to demonstrate a statistically significant difference in the presence of epileptiform EEGs in children with ASD and co-morbid epilepsy as compared to those without co-morbid epilepsy. Furthermore, analysis of children with ASD and developmental regression or ASD and language impairment failed to demonstrate statistically significant differences in presence of epileptiform EEG abnormalities. Together these findings suggest that repeat EEGs should be obtained on a case-by-case basis in children with ASD and epileptiform EEG abnormalities in ASD in the absence of convincing epileptic seizures should be cautiously interpreted.
\end{abstract}

\section{Introduction}

Autism Spectrum Disorder (ASD) represents a significant risk factor for epilepsy with an estimated prevalence of epilepsy in patients with ASD ranging from 2- 46\% [1-3]. Clinically, the association between epilepsy and ASD is well characterized in tuberous sclerosis and early infantile epileptic encephalopathies such as West Syndrome. The pathogenesis of epilepsy and ASD are largely unknown, however, the association of these disorders suggests a shared underlying pathophysiology, possibly one of dysfunctional neuronal networks. While there is basic and translational research underway investigating the pathogenesis of both epilepsy and ASD, reliable biomarkers or diagnostic tests are not available to clinicians attempting to tease apart the complex interaction of these disorders.

Electroencephalogram (EEG) is a widely used diagnostic tool for the evaluation of epilepsy and encephalopathy. In ASD, EEG findings vary widely with published frequencies of epileptiform EEG abnormalities in children with ASD ranging from 6-85.8\% [4,5]. Epileptiform discharges represent regions of cortical hyperexcitability, which indicate increased risk for seizures. However, in the absence of clinical seizures, the significance of epileptiform discharges is controversial and unclear. The ambiguity of the relationship between EEG findings and ASD has led to guidelines that discourage screening EEGs at the time of ASD diagnosis in the absence of a clinical suspicion for epilepsy $[6,7]$.

Several studies have investigated the predictive value of epileptiform EEG abnormalities and their correlation to developing epilepsy in patients with ASD. One small study showed an increased risk for clinical epilepsy in children with ASD with frontal epileptiform EEG abnormalities, suggesting that dysregulation in this region could contribute to epileptogenesis in children with ASD [8]. Another retrospective study of 130 patients with ASD concluded that epileptiform EEGs predicted subsequent onset of epilepsy [3]. However, there were still high rates of epileptiform EEGs in the nonepileptic group. Moreover, $18 \%$ of patients with epileptiform discharges did not develop seizures suggesting that EEG has a high false positive rate in prognosticating subsequent development of epilepsy in ASD. Additionally, the epileptic group had significant differences from the non-epileptic group including higher rates of cognitive impairment, a lower level of adaptive functioning, and higher rates of prescribed psychotropic medications. This may indicate that these patients had more severe ASD or that severe epilepsy is associated with more significant autistic features and lower cognitive functioning. These confounders complicate any direct clinical inferences made to the utility of EEG in ASD without clear seizures.

Overall, there is a lack of data regarding evolution of EEG findings and development of epilepsy in children with ASD. Additional data regarding the evolution of EEG findings in children with ASD may lead to better understanding of the correlation between EEG abnormalities, onset of epilepsy, and ASD [1,9]. We present our retrospective review of children with ASD who had serial EEGs for clinical evaluation. We

${ }^{\star}$ Correspondence to: Drew M Thodeson, MD, Department of Pediatrics and Neurology and Neurotherapeutics, Division of Child Neurology, UT Southwestern Medical Center, Dallas, TX 75390, USA, Tel: 214-456-8242; Fax: 214-456-5210; E-mail: drew.thodeson@utsouthwestern.edu

Key words: autism, ASD, electroencephalogram, EEG, epilepsy

Received: June 13, 2018; Accepted: June 28, 2018; Published: July 05, 2018 
specifically sought to define the prognostic value of EEG and its relation to presence of clinical seizures, language impairment, and history of developmental regression.

\section{Methods}

The study population was identified by querying the neurophysiology database at Children's Health Dallas for patients with the clinical diagnosis of ASD who had two or more EEGs between January 2002 and May 2015. All patients met clinical criteria for the diagnosis of ASD as per the Diagnostic and Statistical Manual of Mental Disorders (DSMIV or V) or as diagnosed by a clinician [10,11]. Medical records were systematically reviewed specifically focusing on patient demographics, medical history, neurological examinations, EEG findings, and antiseizure medication management. While reviewing medical histories, researchers paid close attention to concurrent diagnosis of clinical epilepsy, the presence of profound language impairment, and history of developmental regression. Clinical epilepsy was defined as children with EEG confirmed seizures, a diagnosed clinical epilepsy syndrome, clinical seizures including convulsive seizures, focal seizures with or without altered awareness, or other generalized seizures such as atonic, tonic, absence, and myoclonic seizures. Children without spells or staring spells that were distractible or of uncertain significance were defined as the non-epileptic group. Profound language impairment was defined as patients who were nonverbal or only had a few single words. Moderate language impairment was defined as patients who spoke in phrases or short sentences. Normal or near normal language was defined as children with no apparent language deficits on examination. Developmental regression was defined as a loss of developmental milestones such as speech, social interaction, or ability to perform activities of daily living per DSM-V [10]. All variables, including the diagnosis of epilepsy and ASD was determined at the time of the chart review.

EEGs were evaluated for the presence of epileptiform abnormalities by board certified physicians in clinical neurophysiology or epilepsy. Epileptiform abnormalities were defined as focal, generalized, or multifocal seizures, spike and wave discharges, and sharp waves. Non-epileptiform abnormalities were defined as focal, generalized, or multifocal theta or delta slowing, posterior background slowing, frontal intermittent rhythmic delta activity (FIRDA), and occipital intermittent rhythmic delta activity (OIRDA). Epileptiform activity was coded as a dichotomous variable for each EEG. Initial EEG findings were compared to subsequent EEG and last EEG findings using Chi-square analysis. Initial EEG and subsequent EEG findings were analyzed using Chisquare, Wilcoxan Rank Sum test, and logistic regression to investigate association between EEG findings and both language impairment and developmental regression.

Descriptive analysis of the continuous and categorical data was performed using means, confidence intervals, proportions, and frequencies. Chi-square and Wilcoxon Rank Sum tests, as appropriate to the variable's level of measurement and distribution, were used to investigate associations and differences between sub-groups. Multiple logistic regression was used to model binary outcome based on multiple prognostic factors. The statistical analyses were performed with SAS 9.4 .

\section{Results}

\section{Demographics}

There were 108 patients with ASD were identified with 331 EEGs performed. $71 \%$ of the patients were male and $58 \%$ were diagnosed with epilepsy. $17 \%$ had history of developmental regression (Table 1). The median age of onset of seizures was between five and six years of age (Figure 1).

\section{Epileptiform discharges in ASD}

There was a significant association between an initial EEG outcome and subsequent EEG outcomes $(\mathrm{p}<0.0001) .61 .8 \%$ of patients with initial epileptiform EEG continued to have epileptiform EEGs on subsequent studies. $83 \%$ of patients with initial non-epileptiform EEG continued to have non-epileptiform EEGs on subsequent studies. In addition, treatment with anti-seizure medication did not significantly influence subsequent EEG outcome ( $p>0.05)$. There was no significant difference in the presence of epileptiform EEGs in children with comorbid ASD and epilepsy (35/63 or 55.6\%) as compared to those without epilepsy (20/44 or $45.6 \%)(\mathrm{p}=0.2199)$. No significant association was found between an initial epileptiform EEG and developmental regression ( $\mathrm{p}=0.50)$. Eight children with initial epileptiform EEGs had developmental regression representing $16 \%(8 / 50)$ of all children with initial epileptiform EEGs. Ten children with non-epileptiform EEGs

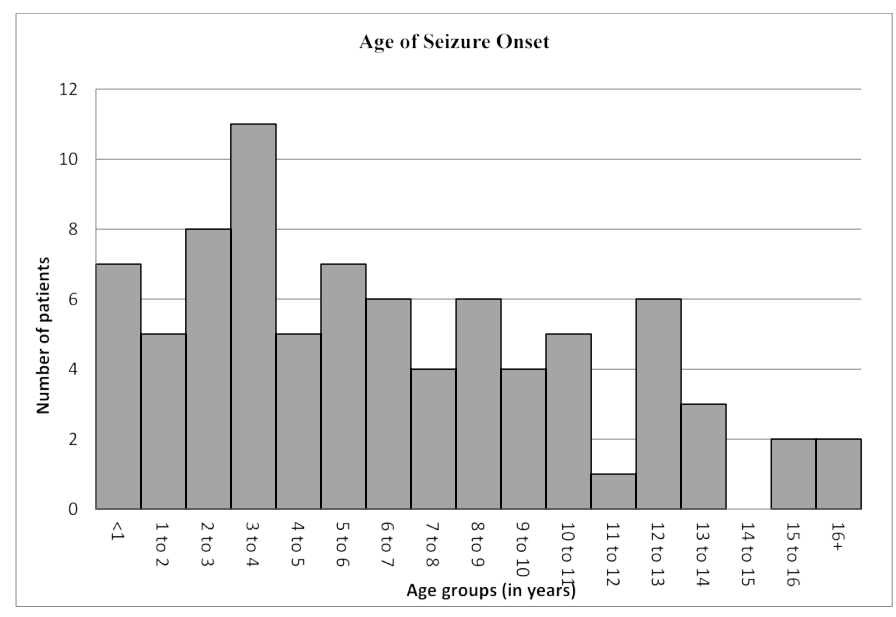

Figure 1. Age of seizure onset

Table 1. Demographics

\begin{tabular}{|c|c|}
\hline Total Patients & 108 \\
\hline Gender & 77 male \\
\hline $\begin{array}{c}\text { Average age at diagnosis of } \\
\text { Autism }\end{array}$ & $\begin{array}{c}2.9 \text { years }(\mathrm{N}=62) \\
\text { Data not available in } 46 \text { patients }\end{array}$ \\
\hline \multicolumn{2}{|l|}{ Clinical seizure types } \\
\hline $\begin{array}{l}\text { No seizures or only staring } \\
\text { spells }\end{array}$ & 44 \\
\hline $\begin{array}{l}\text { Complex partial seizures } \\
\text { with or without convulsions }\end{array}$ & 21 \\
\hline $\begin{array}{l}\text { Convulsions or generalized } \\
\text { seizures (i.e. absence, tonic, } \\
\text { myoclonic) }\end{array}$ & 42 \\
\hline Data not available & 1 \\
\hline Average age of seizure / spell onset & $\begin{array}{l}6 \text { years ( } 1 \text { week }-16 \text { years) } \\
\text { No spells or seizures in } 20 \text { patients } \\
\text { Data not available in } 4 \text { patients }\end{array}$ \\
\hline History of regression & $\begin{array}{c}18 \\
\text { Data not available in } 11 \text { patients }\end{array}$ \\
\hline \multicolumn{2}{|l|}{ Language skills } \\
\hline Nonverbal & 44 \\
\hline Single words & 28 \\
\hline Sentences & 18 \\
\hline Normal / mild impairment & 9 \\
\hline Data not available & 9 \\
\hline
\end{tabular}


had developmental regression representing $21.3 \%$ (10/47) of all children with initial non-epileptiform EEGs. Finally, there was no significant difference in the presence of epileptiform EEGs in children with ASD in relation to language impairment. 59.7\% (43/72) of children with severe language impairment had an initial epileptiform EEG. Whereas, $40.7 \%$ $(11 / 27)$ of children with mild language impairment or normal language development had an initial epileptiform EEG ( $p=0.2572)$.

\section{Discussion}

\section{Initial EEG is a significant prognosticator of subsequent EEG findings}

In our study, there was a significant association between initial EEG findings and subsequent findings $(\mathrm{p}<0.0001)$. Consistent with prior publications, children with ASD who have epileptiform EEG abnormalities on initial EEG are more likely to continue to have epileptiform abnormalities on subsequent EEGs, while children with ASD who have non-epileptiform initial EEGs are more likely to have subsequent non-epileptiform EEGs [12]. After an initial non-epileptiform EEG, $17 \%$ of patients with ASD had a subsequent epileptiform EEG. This suggests that repeat EEGs may have limited utility and should be considered on a clinical basis.

\section{Epileptiform discharges in ASD}

Although children with ASD and epilepsy had a higher occurrence of epileptiform EEGs (55.6\%) as compared to children with ASD without epilepsy $(45.6 \%)$, this difference was not statistically significant. A possible confounder is that our cohort had a high occurrence of epileptiform EEG abnormalities due to referral bias, as the reason for many of the children referred for multiple EEGs was likely due to a strong clinical suspicion for epileptic seizures. Additionally, it is possible that the study is underpowered to see a $10 \%$ difference between the two populations. Perhaps expanding the scope of this study to include more participants or addition of a control group (such as ASD with single EEG) could have unveiled a statistically significant association. Regardless, our data suggests that an abnormal EEG may not be a clear prognostic biomarker for epilepsy in patients with ASD. Utilization of video EEG monitoring, reviewing caregiver's video recording of spells, and careful observation and documentation of spells concerning for seizures must play a critical role in making clinical diagnoses of epilepsy in children with ASD.

Several factors including age of onset of epilepsy, sleep/wake state, presence of specific epilepsy syndromes, and comorbid conditions contribute to the variable sensitivity of interictal EEGs in children with ASD. Although epilepsy is known to have a bimodal distribution of onset in ASD [13] the median age of epilepsy onset in our sample was 6 years old, seemingly skewed to the younger age of seizure or spell onset. Therefore, the age distribution of our sample may overestimate the presence of interictal epileptiform discharges (IEDs) observed in children with ASD and epilepsy as compared to the prevalence in the true ASD population. EEGs that include recordings in the sleep state are more sensitive for IEDs as compared to recordings in the wake state only. Additionally, certain epilepsy syndromes associated with ASD, such as Lennox-Gastaut Syndrome, are associated with higher frequencies of IEDs as compared to non-syndromic epilepsy. Our study did not control for sleep/wake state or epilepsy syndrome, which could confound our results and may explain our high rates of epileptiform discharges.

\section{Developmental regression and epileptiform EEG abnormalities}

We found no association between developmental regression and initial epileptiform EEG abnormalities $(p=0.50)$. Regression is a salient feature of ASD thought to be a risk factor for the development of epilepsy with conflicting evidence [5,10,14-17]. A retrospective review of 889 children with primary ASD failed to show an increased occurrence in sleep epileptiform EEG abnormalities in children with history of regression as compared to those without regression [5]. However, a 2017 meta-analysis concluded that there might be a weak relationship between history of regression and epileptiform EEG abnormalities [17]. It is possible that our cohort was not sufficiently powered to reveal an association between regression and epileptiform EEG abnormalities in children with ASD, however, inconsistencies and lack of clear consensus indicate a need for further research in this area.

\section{Speech impairment and epileptiform EEG abnormalities}

Children with profound speech impairment had a higher rates of epileptiform abnormalities (59.7\%) as compared to those with normal or near normal speech $(40.7 \%)$, but this difference did not meet statistical significance $(\mathrm{p}=0.2572)$. Syndromes such as LandauKleffner syndrome (LKS) and continuous spike wave of sleep (CSWS) suggest an association between language regression and frequent IEDs in sleep. However, it is unclear if IEDs are causal of language impairments in these disorders. Similarly, ASD is characterized by social and language dysfunction and one may hypothesize that higher IED burden in children with ASD may cause language dysfunction [18]. Alternatively, high IED burden and severe language impairment may be epiphenomenon indicative of greater neuronal dysfunction. ASD is a heterogenous disorder with multiple etiologies including idiopathic, structural brain abnormalities, genetic mutations, and metabolic disorders. Investigation of ASD using new technologies including advanced neuroimaging, whole exome, and whole genome sequencing are beginning to define causes of ASD. Differentiating molecular phenotypes and correlating clinical data in the future may further our understanding of the complex interaction between language dysfunction and IEDs in children with ASD.

\section{Conclusion}

Epileptiform EEG abnormalities are common in children with ASD. In our cohort, there was no significant differences of epileptiform EEG abnormalities in children with or without the clinical diagnosis of epilepsy. This supports the recommendation guidelines against routine screening EEG for newly diagnosed ASD [7] as many children with ASD may have an epileptiform EEG without clinical seizures. Not surprisingly, initial EEG was a significant prognosticator of future EEG findings, suggesting that serial EEGs in children with ASD should be obtained on a clinical basis. We demonstrated no difference in epileptiform EEGs in patients with severe language dysfunction or history of developmental regression. The significance of epileptiform EEG abnormalities in children with ASD is unclear and careful clinical correlation is required while making treatment decisions. ((more broad concluding final remark)).

\section{Acknowledgements}

Special thanks to Children's Health Children's Medical Center Dallas clinical research department for their support with statistical analysis. 


\section{Financial support}

Research reported in this publication was supported by the National Center for Advancing Translational Sciences of the National Institutes of Health under award Number UL1TR001105 (D.M.T). The content is solely the responsibility of the authors and does not necessarily represent the official views of the NIH.

\section{References}

1. El Achkar CM, Spence SJ (2015) Clinical characteristics of children and young adults with co-occurring autism spectrum disorder and epilepsy. Epilepsy Behav 47: 183-190. [Crossref]

2. Tuchman R, Rapin I (2002) Epilepsy in autism. Lancet Neurology 1: 352-358.

3. Hara H (2007) Autism and epilepsy: a retrospective follow-up study. Brain Dev 29: 486-490. [Crossref]

4. Yasuhara A (2010) Correlation between EEG abnormalities and symptoms of autism spectrum disorder (ASD). Brain Dev 32: 791-798. [Crossref]

5. Chez MG, Krasne CM, Coughlan C, Kominsky M, Schwartz A (2006) Frequency of epileptiform EEG abnormalities in a sequential screening of autistic patients with no known clinical epilepsy from 1996 to 2005. Epilepsy Behav 8: 267-271. [Crossref]

6. Filipek PA, Accardo PJ, Ashwal S, Baranek GT, Cook EH, et al. (2000) Practice parameter: Screening and diagnosis of autism: Report of the Quality Standards Subcommittee of the American Academy of Neurology and the Child Neurology Society. Neurology 55: 468-479. [Crossref]

7. Kagan-Kushnir T, Roberts SW, Snead OC (2005) Screening Electroencephalograms in Autism Spectrum Disorders: Evidence-Based Guideline. J Child Neurol 20: 197-206. [Crossref]
8. Kanemura H, Sano F, Tando T, Sugita K, Aiharab M. (2013) Can EEG characteristics predict development of epilepsy in autistic children?. Eur J Paediatr Neurol 17: 232237.

9. Ghacibeh GA, Fields C (2015) Interictal epileptiform activity and autism. Epilepsy Behav 47: 158-162. [Crossref]

10. American Psychiatric Association (2013) Diagnostic and statistical manual of mental disorders : DSM-5. 5th edition. Arlington, VA.

11. American Psychiatric Association (2000) Diagnostic and Statistical Manual of Mental Disorders: DSM-IV-TR. 4th ed. Washington, DC.

12. Pillai J, Sperling MR (2006) Interictal EEG and the Diagnosis of Epilepsy. Epilepsia 47: 14-22. [Crossref]

13. Tuchman R, Alessandri M, Cuccaro M (2010) Autism spectrum disorders and epilepsy: moving towards a comprehensive approach to treatment. Brain Dev 32: 719-730. [Crossref]

14. Canitano R, Luchetti A, Zappella M (2005) Epilepsy, electroencephalographic abnormalities, and regression in children with autism. J Child Neurol 20: 27-31. [Crossref]

15. Baird G, Robinson RO, Boyd S, Charman T (2006) Sleep electroencephalograms in young children with autism with and without regression. Dev Med Child Neurol 48: 604-608. [Crossref]

16. Ayta S, Gurses C, Bilgen ZT, Eraksoy M (2016) The clinical and electroencephalography findings of children with pervasive developmental disorder. Turk Noroloji Dergisi 22 : $167-176$

17. Barger BD, Campbell J, Simmons C (2017) The relationship between regression in autism spectrum disorder, epilepsy, and atypical epileptiform EEGs: A meta-analytic review. J Intel Dev Dis 42: 45-60.

18. Trauner DA (2015) Behavioral correlates of epileptiform abnormalities in autism Epilepsy Behav 47: 163-166. [Crossref]

Copyright: $\ 2018$ Thodeson DM. This is an open-access article distributed under the terms of the Creative Commons Attribution License, which permits unrestricted use, distribution, and reproduction in any medium, provided the original author and source are credited. 